\title{
Culture of Puccinia melanocephala on detached leaves and uprights of sugarcane in Puerto Rico ${ }^{1}$
}

\author{
Lii-Jang Liu $^{2}$
}

\begin{abstract}
Sugarcane rust ( Puccinia melanocephala) was cultured through its uredial and telial stages on detached leaves and uprights of 10 sugarcane cultivars in test tube, as well as in petri dish-benzimidazole, kinetin and nutrient solutions. The infection type, incubation period and severity of rust varied with cultivars and with age of the plant. Detached uprights in test tubes containing benzimidazole nutrient solution $(5 \mathrm{ml}$ of $25 \mathrm{p} / \mathrm{m}$ benzimidazole + $5 \mathrm{ml}$ of nutrient solution) stayed green more than 30 days. Uredial and telial pustules developed better on detached uprights than on detached leaves. Rust developed better on detached leaves in test tube containing benzimidazole nutrient solution than on leaves in the petri dishes containing the same solution; there was less rust development on detached leaves from 6-monthold plants than on those from younger ones. More than $95 \%$ of the 90 cultivars inoculated with the detached upright technique, showed rust reactions corresponding with those observed under natural infection in the fields.
\end{abstract}

\section{INTRODUCTION}

Sugarcane rust caused by Puccinia melanocephala H. Syd. \& P. Syd. has become a damaging disease and is affecting the local sugarcane breeding program (7). Approximately $50 \%$ of the parental material is susceptible to the rust ${ }^{3}$. Introduction of additional germplasm for rust resistance, as well as a reliable technique for screening thousands of seedlings, is urgently needed.

Detached-leaf techniques have been widely used to study life cycle (6), physiologic race identification $(4,5,10)$, genetics (10), host parasite relationship $(11,12)$ and routine culture maintenance $(3,9)$ of wheat stem rust caused by Puccinia graminis $\mathrm{f}$. sp. tritici. These techniques with some modifications could be useful for screening thousands of sugarcane seedlings for rust resistance. This study was undertaken to determine whether detached leaf and upright techniques might be used to evaluate sugarcane for rust resistance under local conditions.

\section{MATERIALS AND METHODS}

Tests were performed with PR 62-285, PR 63-1165, PR 64-2705, PR 673129, PR 70-3413, PR 76-1070, PR 78-71, B 4362, Co 798, and Q 90. The

${ }^{1}$ Manuscript submitted to Editorial Board December 1, 1980.

${ }^{2}$ Phytopathologist, Department of Crop Protection, Agricultural Experiment Station, College of Agricultural Sciences, University of Puerto Rico, Rio Piedras, P. R. 00928. Supported in part by the TAD (Tropical Agricultural Development) research grant, USDA Cooperative Agreement No. 58-7B30-9-115).

${ }^{3} \mathrm{Chu}, \mathrm{T}$. L., personal communication. 
plants of these susceptible cultivars were inoculated with urediospores of P. melanocephala, collected at the Lajas and Gurabo Substations.

The sugarcanes were grown in $8 \times 4$ inches black plastic bags in a Saran-tent at $28^{\circ}$ to $32^{\circ} \mathrm{C}$. The plants were kept under routine watering and fertilization regimes. When experiments were established, one of the two upper leaves was removed, fastened to a plate and sprayed with a suspension of urediospores. Approximately 50 spores per $10 \mathrm{X}$ power microscope field were deposited on the leaves. After inoculation, the leaves were again sprayed with water and individually dropped, with cut end down, into a 11 1/2 inches long Pyrex test tube containing $10 \mathrm{ml}$ of benzimidazole, or kinetin or nutrient solution (2) or various combinations of benzimidazole with either kinetin or nutrient solution. Tap water was used for the controls. The test tubes containing the leaves were placed in a dark moist chamber for 24 hours, and then, placed in 8 hours-day chambers for the duration of the experiments.

Leaves were always cut to a length of about $25 \mathrm{~cm}$ long and inoculated on the lower surface. In the case of detached uprights the same procedures were followed. Urediospores of the rust fungus produced on PR 67-3129 were stored in test tubes at about $4^{\circ} \mathrm{C}$ until used, usually not more than 15 days after collection. The modified Cobb scale (8) was used to estimate the rust intensity. Infection type was based on a modified key described by Stakman and Stewart (13). Data on rust severity, infection type and incubation period (the time from inoculation to the appearance of flecks), were recorded. Tests were repeated several times.

\section{EFFECT OF BENZIMIDAZOLE, KINETIN AND NUTRIENT SOLUTION}

In several trials, four leaves from 30-day-old sugarcane plants of each cultivar were inoculated and placed in test tubes containing one of the following solutions: 1) benzimidazole $25 \mathrm{p} / \mathrm{m}$; 2) kinetin $20 \mathrm{p} / \mathrm{m}$ in $5 \%$ sucrose; 3) nutrient solution; 4) benzimidazole + kinetin (1:1); 5) benzimidazole + nutrient solution $(1: 1) ; 6)$ kinetin + nutrient solution (1:1); and 7) tap water. The same experiment was repeated with four detached uprights instead of leaves.

\section{EFFECT OF PLANT AGE}

Sugarcane plants of the 10 cultivars were grown in the Saran tent for 30,90 and 180 days. Then, the leaves were detached, inoculated and placed in the benzimidazole nutrient solution with the same method described above. Not all the tests were made at the same time, but rather when the plants had reached the desired age. Two tests were made, each with four replicate plants per treatment. 


\section{EFFECT OF TEMPERATURE}

Leaves of 30-day-old plants grown in the Saran tent were detached and placed in benzimidazole nutrient solution in test tubes. The test tubes containing the leaves were placed in controlled chambers at 20,24 and $28^{\circ} \mathrm{C}$. There were two trials with four replicate leaves per treatment in each trial.

\section{INOCULATION STUDIES}

A total of 90 cultivars were inoculated by the detached upright inoculation technique. Rust reactions were recorded 10 days after inoculation. Rust reactions of these same cultivars were also recorded under natural infection in the fields for comparison purposes.

\section{RESULTS}

\section{EFFECT OF BENZIMIDAZOLE, KINETIN AND NUTRIENT SOLUTION}

Benzimidazole led to more intense rust development on sugarcane than kinetin. Sugarcane leaves in kinetin solution turned brownish yellow after 3 days. Nutrient solution, however, appeared superior to others in accelerating uredial pustule development. Leaves in nutrient solution stayed greener than those in other solutions; however, contamination of the nutrient solution by fungi was also considerably higher. A combination of benzimidazole with nutrient solution $(5 \mathrm{ml}$ of $25 \mathrm{p} / \mathrm{m}$ benzimidazole +5 $\mathrm{ml}$ of nutrient solution) appeared to be the best treatment for preserving the detached cane leaves for rust development (table 1). The detached upright in the same solution stayed greener longer than the detached leaves. Furthermore, detached leaves in test tube containing the benzimidazole nutrient solution stayed greener longer than those in petri dishes containing the same solution. Uredial pustules appeared on the detached uprights 7 days after inoculation. Telia appeared on the same pustules approximately 3 to 4 weeks after inoculation.

\section{EFFECT OF PLANT AGE}

Uredial pustules developed on each of the leaves that were taken from 30- to 90-day-old plants, but only traces of rust formed on leaves that were obtained from 180-day-old plants. The severity of the rust on the leaves from 180-day-old plants was about 30\% less than that on the leaves from 30-day-old plants. The infection types were also considerably lower on leaves from 180-day-old plants than from 30-day-old plants (table 2). The incubation period (the time needed for flecks to form on inoculation leaves) was 1 week longer on leaves that were detached from 180-day-old plants than on those from the 30-day-old plants. 
TABLE 1.-Effect of benzimidazole, kinetin and nutrient solution on infection types and severity of rust (Puccinia melanocephala) on leaves detached from 30 days-old sugarcane plants ${ }^{1}$

\begin{tabular}{|c|c|c|c|c|c|c|c|c|c|c|c|c|c|c|}
\hline \multirow{2}{*}{$\begin{array}{l}\text { Sugarcane } \\
\text { cultivar }\end{array}$} & \multicolumn{2}{|c|}{$\begin{array}{l}\text { Benzimidazole } \\
\text { alone }\end{array}$} & \multicolumn{2}{|c|}{ Kinetin } & \multicolumn{2}{|c|}{ Nutrient solution } & \multicolumn{2}{|c|}{$\begin{array}{c}\text { Benzimidazole + } \\
\text { kinetin }\end{array}$} & \multicolumn{2}{|c|}{$\begin{array}{l}\text { Benzimidazole + } \\
\text { nutrient solution }\end{array}$} & \multicolumn{2}{|c|}{$\begin{array}{c}\text { Kinetin + nutrient } \\
\text { solution }\end{array}$} & \multicolumn{2}{|c|}{ Tap water } \\
\hline & $\begin{array}{l}\text { Infec- } \\
\text { tion } \\
\text { type }\end{array}$ & $\begin{array}{l}\text { Severity } \\
(\%)\end{array}$ & $\begin{array}{c}\text { Infec- } \\
\text { tion } \\
\text { type }\end{array}$ & $\begin{array}{l}\text { Severity } \\
(\%)\end{array}$ & $\begin{array}{c}\text { Infec- } \\
\text { tion } \\
\text { type }\end{array}$ & $\begin{array}{l}\text { Severity } \\
(\%)\end{array}$ & $\begin{array}{l}\text { Infec- } \\
\text { tion } \\
\text { type }\end{array}$ & $\begin{array}{l}\text { Severity } \\
(\%)\end{array}$ & $\begin{array}{c}\text { Infec- } \\
\text { tion } \\
\text { type }\end{array}$ & $\begin{array}{c}\text { Severity } \\
(\%)\end{array}$ & $\begin{array}{l}\text { Infec- } \\
\text { tion } \\
\text { type }\end{array}$ & $\begin{array}{l}\text { Severity } \\
(\%)\end{array}$ & $\begin{array}{c}\text { Infec- } \\
\text { tion } \\
\text { type }\end{array}$ & $\begin{array}{c}\text { Severity } \\
(\%)\end{array}$ \\
\hline PR 62-285 & 7 & 30 & 6 & 28 & 7 & 31 & 6 & 25 & 7 & 35 & 7 & 30 & 6 & 25 \\
\hline PR 63-1165 & 9 & 40 & 8 & 35 & 9 & 42 & 8 & 30 & 9 & 45 & 6 & 30 & 7 & 25 \\
\hline PR 64-2705 & 8 & 35 & 7 & 30 & 8 & 38 & 7 & 25 & 8 & 30 & 6 & 25 & 6 & 20 \\
\hline PR 67-3129 & 7 & 30 & 6 & 20 & 8 & 50 & 6 & 30 & 8 & 55 & 6 & 25 & 6 & 25 \\
\hline PR 70-3413 & 8 & 35 & 7 & 30 & 8 & 40 & 7 & 20 & 9 & 38 & 7 & 30 & 7 & 25 \\
\hline PR $76-1070$ & 8 & 35 & 7 & 32 & 8 & 38 & 7 & 32 & 9 & 35 & 7 & 20 & 7 & 20 \\
\hline PR 78-71 & 8 & 35 & 7 & 25 & 9 & 55 & 7 & 35 & 9 & 60 & 7 & 30 & 7 & 30 \\
\hline B 4362 & 9 & 40 & 8 & 35 & 9 & 40 & 8 & 34 & 9 & 40 & & 30 & 7 & 35 \\
\hline Co 798 & 8 & 34 & 7 & 30 & 8 & 40 & 7 & 30 & 8 & 35 & 6 & 30 & 6 & 30 \\
\hline Q 90 & 6 & 25 & 5 & 20 & 7 & 45 & 5 & 25 & 7 & 55 & 6 & 25 & 5 & 20 \\
\hline
\end{tabular}

${ }^{1}$ Twenty five micrograms benzimidazole and 20 micrograms of kinetin per ml water. Data based on four detached leaves in each of seven trials. Infection types according to Liu (7) and severity according to Peterson et al. (8). 


\section{EFFECT OF TEMPERATURE}

The length of incubation period varied with cultivars and with temperatures. The incubation period was longer for Co 798 and Q 90 than for the other cultivars and the period was longer at $20^{\circ} \mathrm{C}$ than at $28^{\circ} \mathrm{C}$ for all the cultivars. The severity of rust on individual cultivars varied with the temperatures used in this study (table 3 ).

\section{INOCULATION STUDIES}

Results obtained from upright inoculation studies indicate that more than $95 \%$ of the inoculated cultivars showed rust reactions corresponding to those observed under natural infection in the fields (table 4).

TABLE 2.-Effect of age of plant leaf detachment on rust development on sugarcane cultivars in benzimidazole nutrient solution ${ }^{1}$

\begin{tabular}{|c|c|c|c|c|c|c|}
\hline \multirow{3}{*}{$\begin{array}{l}\text { Sugarcane } \\
\text { cultivars }\end{array}$} & \multicolumn{6}{|c|}{ Rust development on leaves detached from plants at } \\
\hline & \multicolumn{2}{|c|}{1 month old } & \multicolumn{2}{|c|}{3 months old } & \multicolumn{2}{|c|}{6 months old } \\
\hline & $\begin{array}{c}\text { Infection } \\
\text { type }\end{array}$ & $\begin{array}{c}\text { Severity } \\
(\%)\end{array}$ & $\begin{array}{l}\text { Infection } \\
\text { type }\end{array}$ & $\begin{array}{l}\text { Severity } \\
\text { (\%) }\end{array}$ & $\begin{array}{l}\text { Infection } \\
\text { type }\end{array}$ & $\begin{array}{l}\text { Severity } \\
\text { (\%) }\end{array}$ \\
\hline PR $62-285$ & 7 & 30 & 7 & 25 & 4 & 15 \\
\hline PR 63-1165 & 9 & 40 & 9 & 35 & 6 & 10 \\
\hline PR 64-2705 & 8 & 35 & 8 & 30 & 5 & 10 \\
\hline PR 67-3129 & 7 & 35 & 7 & 30 & 5 & 10 \\
\hline PR 70-3413 & 8 & 30 & 8 & 25 & 6 & 10 \\
\hline PR 76-1070 & 8 & 35 & 8 & 32 & 5 & 5 \\
\hline PR 78-71 & 9 & 40 & 9 & 35 & 5 & 10 \\
\hline B 4362 & 9 & 40 & 9 & 35 & 4 & 10 \\
\hline Co 798 & 8 & 35 & 8 & 30 & 4 & 5 \\
\hline Q 90 & 6 & 25 & 6 & 25 & 3 & 5 \\
\hline
\end{tabular}

${ }^{1}$ Five $\mathrm{ml}$ of $25 \mathrm{p} / \mathrm{m}$ benzimidazole $+5 \mathrm{ml}$ of nutrient solution. Data based on four detached leaves in each of six trials. Infection types according to Liu (7) and severity according to Peterson et al. (8).

TABLE 3.-Effect of temperature on rust development on detached leaves of sugarcane cultivars in benzimidazole nutrient solution ${ }^{1}$

\begin{tabular}{|c|c|c|c|c|c|c|c|c|c|}
\hline \multirow{2}{*}{$\begin{array}{l}\text { Sugarcane } \\
\text { cultivars }\end{array}$} & \multicolumn{3}{|c|}{ Infection type } & \multicolumn{3}{|c|}{ Incubation period (days) } & \multicolumn{3}{|c|}{ Severity $(\%)$} \\
\hline & $20^{\circ} \mathrm{C}$ & $24^{\circ} \mathrm{C}$ & $28^{\circ} \mathrm{C}$ & $20^{\circ} \mathrm{C}$ & $24^{\circ} \mathrm{C}$ & $28^{\circ} \mathrm{C}$ & $20^{\circ} \mathrm{C}$ & $24^{\circ} \mathrm{C}$ & $28^{\circ} \mathrm{C}$ \\
\hline PR 62-285 & 7 & 6 & 7 & 7 & 5 & 4 & 30 & 35 & 30 \\
\hline PR 63-1165 & 9 & 9 & 9 & 7 & 6 & 5 & 40 & 40 & 40 \\
\hline PR 64-2705 & 8 & 8 & 8 & 8 & 7 & 6 & 35 & 35 & 35 \\
\hline PR 67-3129 & 7 & 8 & 7 & 7 & 6 & 6 & 35 & 35 & 35 \\
\hline PR 70-3413 & 8 & 8 & 8 & 7 & 6 & 5 & 30 & 35 & 30 \\
\hline PR 76-1070 & 8 & 8 & 8 & 6 & 5 & 4 & 35 & 35 & 35 \\
\hline PR 78-71 & 9 & 9 & 9 & 7 & 6 & 5 & 35 & 35 & 30 \\
\hline B 4362 & 9 & 9 & 9 & 8 & 7 & 6 & 40 & 45 & 40 \\
\hline Co. 798 & 8 & 8 & 8 & 12 & 8 & 7 & 35 & 30 & 35 \\
\hline Q. 90 & 5 & 7 & 6 & 10 & 7 & 7 & 25 & 25 & 26 \\
\hline
\end{tabular}

${ }^{1}$ Five $\mathrm{ml}$ of benzimidazole $+2 \mathrm{ml}$ of nutrient solution. Data based on four detached leaves/treatment in two trials. 
SUGARCANE RUST CULTURES ON LEAVES, UPRIGHTS

TABLE 4.-Reaction of sugarcane varieties to rust disease in Puerto Rico

\begin{tabular}{|c|c|c|c|c|}
\hline \multirow[b]{2}{*}{ Variety } & \multirow[b]{2}{*}{ Acreage } & \multirow[b]{2}{*}{ Percent } & \multicolumn{2}{|c|}{ Reaction to rust } \\
\hline & & & $\begin{array}{c}\text { Field } \\
\text { observation }\end{array}$ & $\begin{array}{l}\text { Detached upright } \\
\text { inoculation }\end{array}$ \\
\hline PR 980 & 63,380 & 46.3 & $\mathrm{HR}^{1}$ & HR \\
\hline PR 1028 & 19,822 & 14.5 & HR & HR \\
\hline H32-8560 & 7,345 & 5.36 & $\mathrm{R}$ & $\mathrm{R}$ \\
\hline PR 62-258 & 5,711 & 4.17 & $\mathrm{R}$ & $\mathrm{R}$ \\
\hline В 49119 & 4,823 & 3.52 & $\mathrm{R}$ & $\mathrm{R}$ \\
\hline PR 64-1791 & 3,759 & 2.74 & MS & MS \\
\hline PR 1059 & 3,390 & 2.47 & $\mathrm{R}$ & $\mathrm{R}$ \\
\hline PR 1013 & 3,013 & 2.20 & $\mathbf{R}$ & $\mathrm{R}$ \\
\hline PR 1152 & 2,291 & 1.67 & $\mathrm{R}$ & $\mathrm{R}$ \\
\hline PR 61-632 & 2,316 & 1.67 & $\mathrm{R}$ & $\mathrm{R}$ \\
\hline B 42231 & 2,044 & 1.49 & $\mathrm{R}$ & $\mathrm{R}$ \\
\hline PR 1048 & 1,491 & 1.08 & MS & MS \\
\hline B $47-310$ & 1,305 & .95 & $\mathrm{R}$ & $\mathrm{R}$ \\
\hline CP 52-43 & 1,158 & .84 & $\mathrm{R}$ & $\mathrm{R}$ \\
\hline PR 1016 & 976 & .71 & HR & HR \\
\hline $\mathrm{N}:$ Co 310 & 946 & .69 & $\mathrm{R}$ & $\mathrm{R}$ \\
\hline В $37-172$ & 698 & .51 & $\mathrm{R}$ & $\mathrm{R}$ \\
\hline PR 1124 & 673 & .49 & $\mathrm{~S}$ & $\mathrm{~S}$ \\
\hline B $50-112$ & 749 & .54 & MS & MS \\
\hline PR 1002 & 745 & .45 & $\mathrm{R}$ & $\mathrm{R}$ \\
\hline M 336 & 353 & .25 & $\mathrm{R}$ & $\mathrm{R}$ \\
\hline B 41227 & 431 & .31 & HR & HR \\
\hline POJ 2878 & 299 & .21 & $\mathrm{R}$ & $\mathrm{R}$ \\
\hline В 34104 & - & - & HR & $\mathrm{HR}$ \\
\hline CB 44-105 & - & - & HR & HR \\
\hline Сo. 798 & - & - & HS & HS \\
\hline Q. 90 & - & - & HS & HS \\
\hline PR 67-3129 & - & - & HS & HS \\
\hline B 4362 & - & - & HS & HS \\
\hline PR 63-1165 & - & - & HS & HS \\
\hline PR 64-2705 & - & - & HS & HS \\
\hline PR 69-3065 & - & - & MS & MS \\
\hline PR 70-3413 & - & - & HS & $\mathrm{HS}$ \\
\hline PR 62-195 & - & - & $\mathrm{R}$ & $\mathrm{R}$ \\
\hline PR 62-739 & - & - & $\mathrm{R}$ & $\mathrm{R}$ \\
\hline PR 63-192 & - & - & MS & MS \\
\hline PR 63-525 & - & - & MR & MS \\
\hline PR 64-245 & - & - & $\mathrm{R}$ & $\mathrm{R}$ \\
\hline PR 64-1548 & - & - & $\mathrm{HR}$ & HR \\
\hline PR 64-1618 & - & - & $\mathrm{HR}$ & HR \\
\hline PR 65-109 & - & - & $\mathrm{S}$ & $\mathrm{R}$ \\
\hline PR 65-153 & - & - & HR & $\mathrm{HR}$ \\
\hline PR 65-413 & - & - & MR & MR \\
\hline PR 65-491 & - & - & HR & HR \\
\hline PR 65-551 & - & - & $\mathrm{R}$ & $\mathrm{R}$ \\
\hline PR 65-554 & - & - & $\mathrm{R}$ & $\mathrm{R}$ \\
\hline PR 65-2638 & - & - & MS & MS \\
\hline
\end{tabular}


TABLE 1-Continued

\begin{tabular}{|c|c|c|c|c|}
\hline \multirow[b]{2}{*}{ Variety } & \multirow[b]{2}{*}{ Acreage } & \multirow[b]{2}{*}{ Percent } & \multicolumn{2}{|c|}{ Reaction to rust } \\
\hline & & & $\begin{array}{c}\text { Field } \\
\text { observation }\end{array}$ & $\begin{array}{l}\text { Detached upright } \\
\text { inoculation }\end{array}$ \\
\hline PR 66-1240 & - & - & MR & MR \\
\hline PR 66-2281 & - & - & $\mathrm{R}$ & $\mathrm{R}$ \\
\hline PR 67-110 & - & - & MR & $\mathrm{MR}$ \\
\hline PR 67-245 & - & - & $\mathrm{R}$ & $\mathrm{R}$ \\
\hline PR 67-406 & - & - & MS & MS \\
\hline PR 67-1070 & - & - & HR & HR \\
\hline PR 67-1355 & - & - & MS & MS \\
\hline PR 67-2295 & - & - & MS & $\mathrm{R}$ \\
\hline PR 67-2467 & - & - & $\mathrm{R}$ & $\mathrm{R}$ \\
\hline PR 67-3052 & - & - & MS & MS \\
\hline PR 68-82 & - & - & HR & $\mathrm{HR}$ \\
\hline PR 68-123 & - & - & $\mathrm{R}$ & $\mathrm{R}$ \\
\hline PR 68-156 & - & - & HR & $\mathrm{HR}$ \\
\hline PR 68-335 & - & - & $\mathrm{R}$ & $\mathrm{R}$ \\
\hline PR 68-1020 & - & - & $\mathrm{R}$ & $\mathrm{R}$ \\
\hline PR 68-1099 & - & - & HR & $\mathrm{HR}$ \\
\hline PR 1216 & - & - & HR & $\mathrm{HR}$ \\
\hline PR 68-2241 & - & - & $\mathrm{R}$ & $\mathrm{R}$ \\
\hline PR 68-2319 & - & - & MR & $\mathrm{HR}$ \\
\hline PR 68-3041 & - & - & $\mathrm{R}$ & $\mathrm{R}$ \\
\hline PR 68-3120 & - & - & $\mathrm{R}$ & $\mathrm{R}$ \\
\hline PR 68-3180 & - & - & $\mathrm{R}$ & $\mathrm{R}$ \\
\hline PR 69-23 & - & - & $\mathrm{R}$ & $\mathrm{R}$ \\
\hline PR 69-42 & - & - & $\mathrm{R}$ & $\mathrm{R}$ \\
\hline PR 69-51 & - & - & $\mathrm{R}$ & $\mathrm{R}$ \\
\hline PR 69-83 & - & - & MS & MS \\
\hline PR 69-222 & - & - & S & $\mathrm{S}$ \\
\hline PR 69-325 & - & - & $\mathrm{R}$ & $\mathrm{R}$ \\
\hline PR 69-455 & - & - & $\mathrm{R}$ & $\mathrm{R}$ \\
\hline PR 69-2030 & - & - & $\mathrm{R}$ & $\mathrm{R}$ \\
\hline PR 69-3061 & - & - & $\mathrm{S}$ & $\mathrm{R}$ \\
\hline PR 69-3065 & - & - & $\mathrm{R}$ & $\mathrm{R}$ \\
\hline PR 69-3081 & - & - & $\mathrm{R}$ & $\mathrm{R}$ \\
\hline PR 69-3172 & - & - & $\mathrm{R}$ & $\mathrm{R}$ \\
\hline PR 69-3329 & - & - & $\mathrm{R}$ & $\mathrm{R}$ \\
\hline PR 70-3391 & - & - & $\mathrm{R}$ & $\mathrm{R}$ \\
\hline PR $70-3413$ & - & - & $\mathrm{R}$ & $\mathrm{S}$ \\
\hline PR 70-3462 & - & - & $\mathrm{R}$ & $\mathrm{R}$ \\
\hline PR 70-3469 & - & - & $\mathrm{R}$ & $\mathrm{R}$ \\
\hline PR 70-3566 & - & - & $\mathrm{R}$ & MS \\
\hline PR 71-358 & - & - & $\mathrm{R}$ & $\mathrm{R}$ \\
\hline PR 71-373 & - & - & $\mathrm{R}$ & $\mathrm{R}$ \\
\hline PR 71-400 & - & - & $\mathrm{R}$ & $\mathrm{R}$ \\
\hline
\end{tabular}

${ }^{1} \mathrm{HR}=$ Highly resistant, $\mathrm{HS}=$ Highly susceptible, $\mathrm{MR}=$ Moderately resistant, $\mathrm{MS}=$ Moderately susceptible, $\mathrm{S}=$ Susceptible, $\mathrm{R}=$ Resistant. 


\section{DISCUSSION}

The results indicate that more than $95 \%$ of the cultivars inoculated via the detached upright technique showed rust reactions corresponding with those observed under natural infection in the field. Thus, detached leaf and upright techniques can be used to evaluate sugarcane cultivars for rust resistance. When the detached-leaf technique is used, it is extremely important to select leaves from plants less than 6-month-old in order to avoid the effect of plant age on rust development. This agrees also with field observations made in Puerto Rico that canes older than 6 months are less susceptible to Puccinia melanocephala.

It was also found that rust development was better on detached leaves in test tubes with adequate air humidity than on leaves in the greenhouse. This finding is in line with those of Browder (3) and Atif and Wilcoxson (1). Since detached uprights could easily be obtained at any age of the plants by topping the main shoot, and since detached uprights of sugarcane stay greener longer in benzimidazole nutrient solution than detached leaves, they should be extremely useful in screening thousands of sugarcane seedlings for rust resistance.

\section{RESUMEN}

En hojas desprendidas y brotes de 10 variedades de caña de azúcar se cultivó la roya de la caña (Puccinia melanocephala) en el teliostadio y la fase urédica. El cultivo se hizo en tubos de ensayo y placas con benzimidazol, cinetina y una solución nutritiva.

El tipo de infección, el período de encubación y la severidad de la roya variaron entre las plantas. Los brotes desprendidos cultivados en tubos de ensayo que contenían una solución nutritiva de benzimidazol ( $5 \mathrm{ml}$ de una solución de $25 \mathrm{ppm}$ más $5 \mathrm{ml}$ de la solución nutritiva) permanecieron verdes por más de 30 días.

Las pústulas urediospóricas y teliospáricas desarrollaron mejor en los brotes desprendidos que en las hojas desprendidas. La roya desarrolló mejor en las hojas desprendidas colocadas en los tubos de ensayo que contenian la solución nutritiva de benzimidazol que en las que se cultivaron en placas con la misma solución. En las hojas desprendidas de plantas de 6 meses, la roya desarrolló menos que en las de plantas más jóvenes.

De las 90 variedades inoculadas mediante la técnica del brote desprendido, más del $95 \%$ presentaron una reacción igual a la observada en el campo en condiciones naturales de infección.

\section{LITERATURE CITED}

1. Atif, A. H. and R. D. Wilcoxson, 1975. Responses of detached tissues of adult wheat plants to Puccinia graminis tritici, Phytopathology 65: 318-21. 
2. Blankendaal, R., H. Hodgson, D. G. Davis, R. A. Haerauf, and R. H. Shimabukuro, 1972. Growing plants without soil for experimental use, USDA-ARS Misc. Publ. No. 1251.

3. Browder, L. E., 1964. A modified detached-leaf culture technique for study of cereal rusts, Plant Dis. Rep. 48: 906-08.

4. Doling, D. A., 1964. The use of benzimidazole for the detached-leaf culture of $P$. striiformis. Pages 55-63 in Cereal Rust Conferences, 1964. Plant Breeding Research Institute, Cambridge, England.

5. Hermansen, J. E., 1964. Identification of physiologic races by means of detached-leaves, Pages 90-91 in Cereal Rust Conferences, 1964. Plant Breeding Research Institute, Cambridge, England.

6. Hooker, A. L. and C. E. Yarwood, 1966. Culture of Puccinia sorghi on detached leaves of corn and Oxalis corniculata, Phytopathology 56: 536-39.

7. Liu, L. J., 1979. Rust of sugarcane in Puerto Rico, Plant Dis. Rep. 63 (4): 256-58.

8. Peterson, R. F., A. B. Campbell, and A. E. Hannak, 1948. A diagrammatic scale for estimating rust intensity on leaves and stems of cereals, Can. J. Res. C 26: 496-500.

9. Prassannakumari, P. and R. D. Wilcoxson, 1970. Detached leaf culture of brown and black rusts of wheat, Indian Phytopathol. 23: 538-40.

10. Prescott, J. M. and H. C. Young, Jr., 1968. A system for rapid classification of cultures of Puccinia recondita f. sp. tritici, Plant Dis. Rep. 52; 437-40.

11. Samborski, D. J., F. R. Forsythe, and C. Person, 1958. Metabolic changes in detached wheat leaves floated on benzimidazole and the effect of these changes on rust reaction, Can. J. Bot. 36: 591-601.

12. Silverman, W., 1960. The development of stem rust on wheat leaves treated with some sugars and sugar alcohols, Phytopathology 50: 114-19.

13. Stakman, E. D., D. M. Stewart, and W. O. Loegering, 1962. Identification of physiologic races of Puccinia graminis var. tritici, USDA, Agric. Res. Serv. Bull. E 617. 